\title{
OTIMIZAÇÃO E VALIDAÇÃO INTRALABORATORIAL DE MÉTODO PARA ANÁLISE DE RESÍDUOS DE CLORANFENICOL EM LEITE CAPRINO POR CROMATOGRAFIA GASOSA COM DETECÇÃO POR CAPTURA DE ELÉTRONS (CG/DCE)
}

\author{
Jaff R. da Silva, Luciana T. Silva e Janice I. Druzian* \\ Departamento de Análises Bromatológicas, Faculdade de Farmácia, Universidade Federal da Bahia, Rua Barão de Geremoabo, \\ s/n, Campus de Ondina, 40170-210 Salvador - BA, Brasil
}

Recebido em 3/2/09; aceito em 21/6/09; publicado na web em 25/11/09

\begin{abstract}
OPTIMIZATION AND INTRALABORATORIAL VALIDATION OF METHOD FOR ANALYSIS OF CHLORAMPHENICOL RESIDUES IN GOAT MILK BY GC/ECD. The presence of chloramphenicol residues in goat milk can cause toxic effects in the population. The present work consists of the optimization and validation of analytical methodology for determination of chloramphenicol residues in goat milk by GC/ECD. The extraction was made with ethyl acetate and the clean-up with SPE-C18. The identification was made by comparison of retention time and GC/MS, and the quantification by external standard. The method was selective, linearity (0.998), precise (5.8-13.4\%), exact (69.87-73.71\%) and robust. The LOD and LOQ of method were 0.030 and $0.10 \mu \mathrm{g} / \mathrm{kg}$, respectively. The method was efficiently for analysis of chloramphenicol in goat milk.
\end{abstract}

Keywords: chloramphenicol residues; goat milk; GC/ECD.

\section{INTRODUÇÃO}

Os antibióticos e outros medicamentos administrados em animais podem caracterizar perigos relacionados ao risco de intoxicações de origem alimentar para os humanos. ${ }^{1-3}$ Devido a isto, o monitoramento dos resíduos de medicamentos veterinários em alimentos tornou-se uma área de preocupação constante dos consumidores, órgãos reguladores e instituições de pesquisa. ${ }^{1-4}$

Na prática veterinária e/ou durante processamento, cloranfenicol (CAP) (Figura 1) pode ser utilizado como antibiótico contra bactérias infecciosas devido ao seu amplo espectro de ação e baixo custo. ${ }^{5-7}$ Entretanto, na terapêutica humana CAP só é utilizado como fármaco de última escolha quando não existe a disponibilidade de outros fármacos eficazes com menor risco toxicológico. Uma concentração plasmática de $25 \mu \mathrm{g} / \mathrm{mL}$ de CAP já possui ação tóxica induzindo aplasia medular e, dependendo do nível e do tempo de exposição e de fatores idiossincráticos, pode culminar em um quadro de anemia aplásica no ser humano. ${ }^{8,9}$

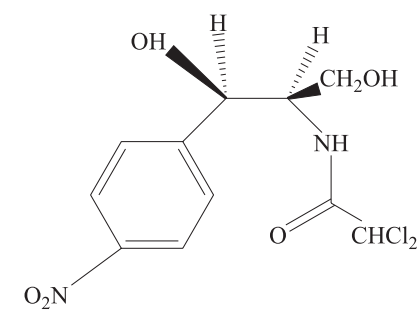

Figura 1. Estrutura química do CAP

Em animais leiteiros, tais como bovinos, caprinos e ovinos, os antibióticos são comumente utilizados para tratamento de mastites, doenças respiratórias e do trato reprodutivo. ${ }^{10}$ Isto está diretamente relacionado com a incidência de resíduos no leite e contribui para o surgimento de bactérias resistentes. ${ }^{10}$ No período de dezembro de 2004 a março de 2006, foram detectados resíduos de antibióticos $\beta$-lactâmicos em 155 amostras de leite caprino originadas na região do Cariri Paraibano. ${ }^{11}$ Em 2007,

*e-mail: druzian@ufba.br foram isoladas de amostras de leite bovino do Rio de Janeiro diversas espécies de enterococos (E. faecalis, E. casseliflavus, E. faccium, E. gallinamm, E. durans e E. gilvus) multirresistentes a antimicrobianos. ${ }^{12}$ Portanto, além do risco de ação tóxica à saúde humana associada a resíduos de CAP em alimentos, a indução de resistência bacteriana constitui um risco adicional à saúde pública. ${ }^{3,13}$ Sendo assim, torna-se necessária a existência de regulamentações e métodos analíticos para promover o monitoramento de resíduos e o controle sanitário desta prática, principalmente em alimentos de elevado consumo, tais como leites. ${ }^{4,10,11}$

Em 2007, a produção total de leite no Brasil foi estimada em 25.464.000 toneladas, sendo que 137 mil toneladas correspondem ao leite caprino. ${ }^{14,15}$ Segundo o Instituto Brasileiro de Geografia e Estatística (IBGE), o rebanho nacional de caprinos em 2007 foi estimado em mais de 9 milhões de cabeças. Neste contexto, a Bahia destacase ocupando o primeiro lugar, seguida de Pernambuco e Piauí. ${ }^{14} \mathrm{O}$ leite caprino tem sido recomendado para crianças, pessoas idosas e convalescentes, devido ao alto valor nutricional, boa digestibilidade e baixo potencial alergênico. ${ }^{16}$ Estas recomendações evidenciam a necessidade de um monitoramento dos resíduos de CAP, assim como o desenvolvimento de métodos eficazes para a determinação deste resíduo na matriz.

A avaliação do Joint FAO/WHO Expert Committe on Food Additives (JECFA), em 2004, concluiu que existem evidências que CAP é genotóxico in vivo, sendo prudente assumir que CAP pode causar alguns efeitos, tais como câncer. O comitê também considera que os estudos epidemiológicos em humanos mostram que o tratamento com CAP está associado com a indução de anemia aplásica, que pode ser fatal. Sendo assim, o comitê concluiu não ser apropriado o estabelecimento de limite máximo de resíduos (LMR) e ingestão diária aceitável (IDA) de CAP, sendo ambos classificados como "não alocado". ${ }^{9}$ Levando este fato em consideração, a Organização Mundial da Saúde (OMS) preconiza que o uso de CAP para fins veterinários seja restrito a animais não destinados à alimentação humana. ${ }^{9}$ Consequentemente, na União Européia (UE), nos Estados Unidos (EUA) e Canadá, o uso de CAP em alimentos não é autorizado. ${ }^{5,9,17}$ No Brasil, a Portaria 448/98 do Ministério da Agricultura, Pecuária e Abastecimento (MAPA) proíbe o uso deste antibiótico em animais destinados à alimentação humana. ${ }^{17} \mathrm{Com}$ o intuito de avaliar o potencial de exposição do consumidor a resíduos 
de medicamentos veterinários (RMV) pela ingestão de alimentos de origem animal, em 2003, a ANVISA criou, através da Resolução RDC $\mathrm{n}^{\circ}$ 253, o Programa Nacional de Análise de Resíduos de Medicamentos Veterinários em Alimentos Expostos ao Consumo (PAMVet). ${ }^{1,17}$

O PAMVet indica o uso das técnicas de CG/DCE e CG/EM para a determinação de resíduos de CAP em leite bovino. ${ }^{1}$ Entretanto, diversas estratégias analíticas para extração e determinação de resíduo de CAP de matrizes alimentícias têm sido recentemente empregadas. ${ }^{18-35}$ Métodos que utilizam a técnica de cromatografia líquida de alta eficiência com detector por ultravioleta (CLAE/UV) detectam níveis de até $4,0 \mu \mathrm{g} / \mathrm{kg} \cdot{ }^{3,18-21}$ Métodos por cromatografia gasosa com detecção por captura de elétrons (CG/DCE) ou pela técnica de espectrometria de massas com ionização química negativa (CG/EM/ IQN) conseguem detectar níveis menores de 1,0 $\mu \mathrm{g} / \mathrm{kg} .{ }^{22-24}$ Métodos que associam a CLAE à técnica de espectrometria de massa-massa em tandem (CL/EM/EM) detectam valores de até $0,020 \mu \mathrm{g} / \mathrm{kg} .{ }^{25-31}$

Os métodos existentes para a análise de resíduo de CAP em leite têm sido desenvolvidos para leite bovino,,$^{3,4,19-21,23,24,26-31,36-48}$ não sendo validados para leite caprino. Portanto, o objetivo do presente estudo foi otimizar e validar, em nível intralaboratorial, um método para a determinação do resíduo de CAP em leite caprino, utilizando as técnicas de cromatografia gasosa com detecção por captura de elétrons (CG/ DCE) e cromatografia gasosa por espectrometria de massas (CG/EM).

\section{PARTE EXPERIMENTAL}

\section{Reagentes e solventes}

O padrão de CAP utilizado foi obtido da Sigma-Aldrich (St. Louis, MO 63103 USA). O acetato de etila, hexano e ciclo-hexano (Mallinckrodt Chemicals, USA) e metanol, acetonitrila e clorofórmio (Mallinkrodt Baker, México) utilizados foram grau pesticida. O cloreto de sódio (Quimex, Brasil) e o sulfato de sódio (Vetec, Brasil) utilizados foram grau p.a. A água foi purificada usando um sistema Milli-Q (Millipore, USA). Os cartuchos de C18 de $500 \mathrm{mg}$ foram adquiridos da J. T. Baker.

\section{Equipamentos cromatográficos}

Foram utilizados um cromatógrafo gasoso CP3800 Varian (CG/ DCE) e software StarWorkstation versão 6.41; e um cromatógrafo gasoso Clarus 500 PerkinElmer (CG/EM), software TurboMass versão 4.5.0 e biblioteca NIST 98; ambos equipados com injetores com e sem divisão de fluxo (split/splitless).

\section{Soluções de CAP}

A solução padrão estoque foi preparada pela derivatização de 1,5 mg do padrão de CAP liofilizado $(24 \mathrm{~h}$ ) com $0,5 \mathrm{~mL}$ do reagente Sigma SIL-A (trimetilclorosilano:hexametildissilazano:piridina; 1:3:9; v/v/v) em frasco âmbar previamente seco em estufa e mantido em dissecador com sílica ativada. A reação se processou por $15 \mathrm{~min}$ a $80^{\circ} \mathrm{C}$ em estufa. $\mathrm{O}$ extrato de CAP derivado foi seco com fluxo de $\mathrm{N}_{2}$ e diluído com 1,5 mL ciclo-hexano:hexano (60:40; v/v), obtendo-se uma solução de 1000 $\mathrm{mg} / \mathrm{L}$ de CAP silanizado. A partir da diluição desta solução, obteve-se uma solução intermediária de $50 \mu \mathrm{g} / \mathrm{L}$. Por diluições sucessivas desta solução com ciclo-hexano:hexano (60:40; v/v), obtiveram-se as soluções padrões nas concentrações de 0,$31 ; 0,62 ; 1,2 ; 2,5$ e $5,0 \mu \mathrm{g} / \mathrm{L}$.

A solução de fortificação para os ensaios de recuperação foi preparada pela diluição do padrão de CAP não silanizado em metanol na concentração de $0,15 \mu \mathrm{g} / \mathrm{mL}$.

As soluções foram acondicionadas em frasco de vidro âmbar, mantidas sob atmosfera inerte $\left(\mathrm{N}_{2}\right)$ e protegidas da luz a $-18{ }^{\circ} \mathrm{C}$, até o momento da análise.

\section{Aquisição de amostras}

Para a otimização e validação do método, foram utilizadas amostras de leite de cabra UHT adquiridas em supermercados de Salvador/ BA. As amostras foram identificadas, registradas e armazenadas a -18 ${ }^{\circ} \mathrm{C}$ até o momento da análise, conforme descrito pelo MERCOSUL e Codex Alimentarius. ${ }^{49,50}$

\section{Extração do analito da matriz}

$\mathrm{O}$ método de extração do resíduo de $\mathrm{CAP}$ foi adaptado à matriz leite caprino com base no método oficial 203/01 do MAPA, que segue as normas do MERCOSUL e Codex Alimentarius. ${ }^{18,49,50}$ Foram preparadas alíquotas de $10 \pm 0,1 \mathrm{~g}$ de amostra. $\mathrm{O}$ analito foi extraído por duas partições consecutivas com $20 \mathrm{~mL}$ de acetato de etila e agitação em vórtex por 6 min, e centrifugação a $4400 \mathrm{rpm}$ a $15^{\circ} \mathrm{C}$ por $3 \mathrm{~min}$ para separar o extrato. O extrato obtido foi evaporado sob fluxo de $\mathrm{N}_{2}$ a $50 \pm 5^{\circ} \mathrm{C}$ até aproximadamente $1 \mathrm{~mL}$, e $5 \mathrm{~mL}$ de solução de $\mathrm{NaCl}$ $4 \%$ foram adicionados, seguidos de agitação em vórtex (1 min). O restante de acetato de etila foi seco com $\mathrm{N}_{2}$. Este resíduo foi lavado 3 vezes com $10 \mathrm{~mL}$ de hexano.

\section{Purificação}

A solução salina obtida foi aplicada em cartucho C18 previamente ativado com $5 \mathrm{~mL}$ de metanol, $5 \mathrm{~mL}$ de clorofórmio, $5 \mathrm{~mL}$ de metanol e $10 \mathrm{~mL}$ de água. Após a aplicação, o cartucho foi lavado com 10 $\mathrm{mL}$ de água e $5 \mathrm{~mL}$ de solução de metanol $20 \%$. O resíduo foi eluído com $3 \mathrm{~mL}$ de acetonitrila para um tubo de vidro.

\section{Derivatização}

$\mathrm{O}$ extrato purificado foi evaporado até a secura com $\mathrm{N}_{2}$ a $50 \pm$ $5{ }^{\circ} \mathrm{C}$ e silanizado com $100 \mu \mathrm{L}$ de trimetilclorossilano:hexametildissilazano:piridina $(1: 3: 9 ; \mathrm{v} / \mathrm{v} / \mathrm{v})$, a $80{ }^{\circ} \mathrm{C}$ por $15 \mathrm{~min}$. Após a derivatização, o extrato silanizado foi seco sob fluxo de $\mathrm{N}_{2}$ a $50 \pm$ $5{ }^{\circ} \mathrm{C}$ e, rapidamente, dissolvido em $0,5 \mathrm{~mL}$ de ciclo-hexano:hexano (60:40; v/v). O extrato de CAP silanizado foi agitado em vórtex por 1 min e centrifugado por $3 \mathrm{~min}$ a $4400 \mathrm{rpm}$ e $15^{\circ} \mathrm{C}$, seguindo para a análise por CG/DCE.

\section{Confirmação da derivatização por CG/EM}

As condições cromatográficas para a identificação conclusiva do CAP silanizado foram: injeção de $1 \mu \mathrm{L}$ no modo de injeção sem divisão de fluxo (splitless) utilizando uma câmara de vaporização com $2 \mathrm{~mm}$ de diâmetro interno; separação em coluna capilar DB-1 (30 m x $0,25 \mathrm{~mm} \times 0,25 \mu \mathrm{m})$ a $80^{\circ} \mathrm{C}$ por $1 \mathrm{~min}$, aumentando a $30^{\circ} \mathrm{C} / \mathrm{min}$ até $230^{\circ} \mathrm{C}$, permanecendo por $14 \mathrm{~min}$, totalizando $20 \mathrm{~min}$ de corrida. $\mathrm{O}$ gás de arraste foi hélio a $1 \mathrm{~mL} / \mathrm{min}$. A fragmentação das moléculas foi obtida através de ionização por impacto de elétrons (EI) com energia de $70 \mathrm{eV}$. O espectrômetro de massas foi programado para varredura na faixa de 50 a $550 \mathrm{~m} / \mathrm{z}$. As temperaturas da linha de transferência e fonte de ionização foram, respectivamente, 200 e $180{ }^{\circ} \mathrm{C}$.

\section{Separação, detecção, identificação por comparação de tempo de retenção $\left(t_{R}\right)$ e quantificação}

O método para separação e determinação do analito por CG/DCE foi otimizado com base no método 203/03 do MAPA, que segue as normas MERCOSUL e USDA. . $2,49,51,52^{2}$

Foi injetado $1 \mu \mathrm{L}$ do extrato silanizado no modo de injeção sem divisão de fluxo (splitless) no CG/DCE. No injetor foi utilizada uma 
câmara de vaporização com $2 \mathrm{~mm}$ de diâmetro interno. A separação foi realizada com coluna capilar DB-1 (60 m x 0,25 mm x 0,25 $\mu \mathrm{m}$ ) sob as seguintes condições: $90{ }^{\circ} \mathrm{C}$ durante 1 min aumentando a 60 ${ }^{\circ} \mathrm{C} / \mathrm{min}$ até $200{ }^{\circ} \mathrm{C}$ e permanecendo por $3 \mathrm{~min}, 5^{\circ} \mathrm{C} / \mathrm{min}$ até atingir $230{ }^{\circ} \mathrm{C}$ e permanecendo por $3 \mathrm{~min}$ e, por fim, aumentando $5{ }^{\circ} \mathrm{C} / \mathrm{min}$ até $260{ }^{\circ} \mathrm{C}$, onde permaneceu por $35,17 \mathrm{~min}$, totalizando $60 \mathrm{~min}$ de corrida. $\mathrm{O}$ gás de arraste utilizado foi hélio a $1 \mathrm{~mL} / \mathrm{min}$. As temperaturas do injetor e detector foram, respectivamente, 280 e $320^{\circ} \mathrm{C}$. A identificação do resíduo foi realizada por comparação de tempo de retenção $\left(t_{R}\right)$ e a quantificação foi realizada por padronização externa.

\section{Procedimentos de validação}

Os ensaios de validação foram realizados por CG/DCE seguindo os parâmetros descritos pelas normas brasileiras e internacionais. $33,34,49,50$

Para a linearidade (padronização externa), foram empregadas soluções de CAP silanizado a 0,31; 0,62; 1,2; 2,5 e 5,0 $\mu \mathrm{g} / \mathrm{L}$, injetadas em triplicata. A origem foi incluída na curva analítica. ${ }^{33,34}$

Foram pesadas alíquotas de $10 \pm 0,1 \mathrm{~g}$ de amostras brancas e estas foram fortificadas, em três repetições, com 20, 100 e $200 \mu \mathrm{L}$ da solução de fortificação de $0,15 \mu \mathrm{g} / \mathrm{mL}$, equivalentes a contaminações de 0,$30 ; 1,5$ e $3,0 \mu \mathrm{g} / \mathrm{kg}$, respectivamente. Em cada grupo de amostras fortificadas foi incluída uma amostra branca para confirmação da não detecção do analito. Através destes ensaios, foram avaliadas a seletividade, exatidão, precisão e robustez. ${ }^{33,34}$ A precisão foi avaliada em dois níveis: precisão intradia (repetitividade) com triplicatas em três concentrações distintas; e precisão intermediária, tendo como variáveis dois analistas e dois dias distintos também em triplicatas.

Para determinar os limites de detecção (LOD) e quantificação (LOQ) do método, foram realizados ensaios com amostras fortificadas em concentrações decrescentes a partir de $0,30 \mu \mathrm{g} / \mathrm{kg} .{ }^{33,34}$

Uma vez que as variáveis, temperatura de evaporação, massa de amostra, fluxo de nitrogênio durante a secagem e forma de eluição, são definidas pelos métodos de referência, ${ }^{18,22}$ as variáveis adotadas para avaliar a robustez, foram a massa de sulfato de sódio e o tempo de derivatização do extrato.

Os resultados de precisão e robustez obtidos foram avaliados pelo tratamento estatístico da ANOVA.

\section{Critérios de aceitação}

Os critérios adotados como referência para avaliação da exatidão e precisão foram os estabelecidos pelo MERCOSUL e Codex Alimentarius (Tabela 1). ${ }^{49,50}$

Tabela 1. Critérios adotados como referência para exatidão e precisão segundo a faixa de concentração estabelecida pelo Codex Alimentarius e MERCOSUL ${ }^{49,50}$

\begin{tabular}{lcc}
\hline Concentração $(\mu \mathrm{g} / \mathrm{kg}$ ou L) & Exatidão $(\%)$ & Precisão (\%RSD) \\
\hline$\leq 1,0$ & 50 a 120 & 35 \\
1,0 a 10 & 60 a 120 & 30 \\
\hline
\end{tabular}

\section{RESULTADOS E DISCUSSÃO}

Entre 2004-2005, o PAMVet fez o monitoramento de CAP em 306 amostras de leite bovino em pó e UHT coletadas em diversos estados do Brasil por métodos de imunoensaios, encontrando 22 amostras contaminadas. ${ }^{53}$ Para leite caprino, todavia, não foram encontrados estudos de monitoramento e nem métodos desenvolvidos e validados, o que sugere ser este o primeiro estudo.

\section{Extração e purificação do analito da matriz}

A extração do CAP da matriz foi realizada com acetato de etila. O hexano foi o solvente usado para a remoção dos lipídios do extrato. Conforme observado em outros estudos com leite bovino e outras matrizes, ${ }^{20,27,54-56}$ constatou-se também, no presente trabalho, que as etapas de partição com hexano foram suficientes para a purificação do extrato, obtendo-se extratos extremamente límpidos, sem resíduos de gorduras, possibilitando o desenvolvimento de um procedimento simples para a retirada da fração lipídica. A maioria dos métodos existentes para a determinação de resíduos de CAP nas mais diferentes matrizes faz a remoção da fração lipídica da amostras com solventes orgânicos apolares. ${ }^{54}$ Este processo geralmente é realizado utilizando-se, além de hexano, clorofórmio e/ou heptano..$^{27,29,48,54}$ Esta etapa é crucial, pois a presença de gorduras pode reduzir a eficiência do processo de purificação devido à competição entre os lipídios e o CAP pelos sítios ativos da coluna C18.

Na purificação por SPE C18, é importante que as etapas de lavagem e eluição se processem sob ação da gravidade para se obter uma melhor purificação do extrato, resultando em cromatogramas com menor quantidade de interferentes e, principalmente, de coeluentes..$^{22}$ A redução da pressão com o auxílio de uma bomba de vácuo mostrou-se mais eficiente após a lavagem com água, para auxiliar na secagem do cartucho.

Foi constatado também que em todas as etapas de secagem dos extratos a evaporação deve ser feita sob um fluxo brando de $\mathrm{N}_{2}$, e sob constante observação, pois a secagem excessiva pode acarretar a perda do $\mathrm{CAP}^{22}$

\section{Análise qualitativa}

O CG/EM com EI não possui sensibilidade compatível ao CG/ DCE. O DCE é mais sensível, permitindo detectar níveis inferiores a $1,0 \mu \mathrm{g} / \mathrm{kg}$ de CAP. Portanto, nas condições cromatográficas definidas para o CG/EM, foi possível detectar CAP silanizado somente a partir da concentração de $3,0 \mathrm{mg} / \mathrm{mL}$, que é $6 \times 10^{5}$ vezes maior que a maior concentração $(5,0 \mu \mathrm{g} / \mathrm{L})$ usada na curva analítica do CG/ DCE, resultando um $\mathrm{t}_{\mathrm{R}}$ de 13,5 min. Portanto, a identificação do CAP silanizado foi confirmada pela comparação entre o espectro de massa obtido com o espectro da biblioteca NIST. Pela análise do espectro obtido, constatou-se que o método de derivatização do CAP é eficaz, obtendo-se o O,O-Bis-(Tri-Metil-Silil)-Cloranfenicol como produto da silanização (Figura 2).

O padrão de CAP, bem como o extrato da matriz em branco e fortificada, depois de silanizados, foram analisados por CG/DCE. Sob as condições cromatográficas definidas, o valor do $t_{R}$ do CAP silanizado no CG/DCE foi de 36,6 min, e nestas condições nenhum coeluente foi identificado (Figura 3).

\section{Validação do método analítico}

\section{Seletividade}

De acordo com os cromatogramas do branco da matriz, do padrão de CAP silanizado e da matriz fortificada, evidencia-se a seletividade do método, pela ausência da detecção de picos no $t_{R}$ do analito (Figura 3). O método proposto demonstrou ser seletivo, não sendo afetado pelos outros constituintes da matriz ou por interferentes dos reagentes e solventes.

\section{Linearidade}

Para a quantificação empregou-se o método da padronização externa, visando obter análises com menores custo e tempo de análise. A linearidade de um método mostra quanto os resultados obtidos são diretamente proporcionais à concentração do analito, dentro de um intervalo especificado. ${ }^{33}$ 

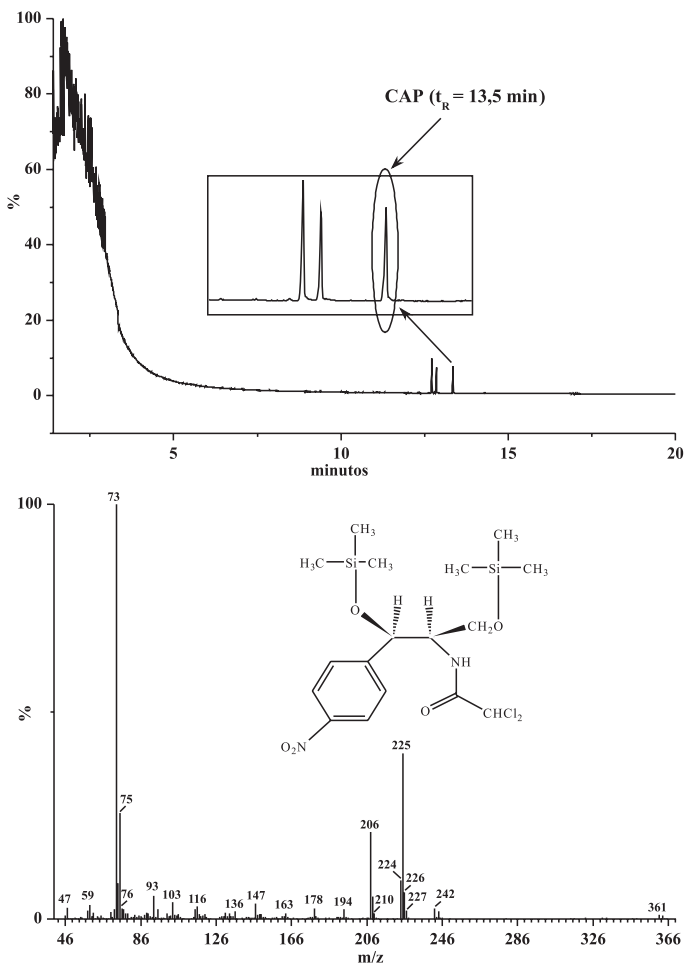

Figura 2. Identificação do CAP silanizado por CG/EM: (A) cromatograma da solução padrão CAP silanizado $(3,0 \mathrm{mg} / \mathrm{mL}) ;(B)$ espectro de massa obtido e estrutura do CAP Bis-silanizado

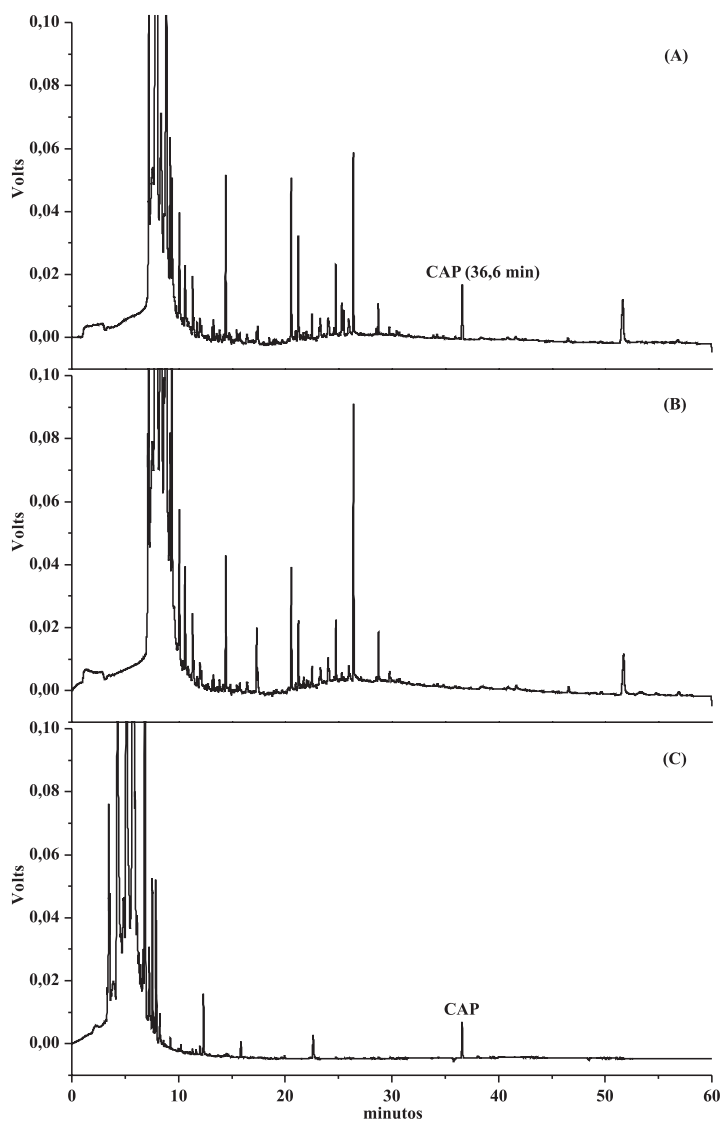

Figura 3. Cromatogramas obtidos por CG/DCE da amostra leite de cabra: (A) amostra fortificada com 1,5 $\mu \mathrm{g} / \mathrm{kg}$ de CAP; (B) amostra em branco; (C) padrão de 1,2 $\mu \mathrm{g} / \mathrm{kg}$ de CAP silanizado
Os valores das áreas dos picos das soluções de CAP silanizado versus as respectivas concentrações foram utilizados para aplicar a regressão linear via método dos mínimos quadrados. A curva analítica obtida por padronização externa com cinco níveis de calibração injetados em triplicata no CG/DCE resultou uma equação de reta (y $=5200,3 \mathrm{x}-382,43)$ com coeficiente de determinação $\left(\mathrm{R}^{2}\right)$ de 0,998 (Figura 4). De acordo com os critérios de aceitabilidade dos resultados, o coeficiente de determinação da curva analítica $\left(\mathrm{R}^{2}\right)$ deve ser 0,995. $\cdot^{33,49,50}$ Os valores de linearidade obtidos por métodos que fazem a quantificação por padronização interna são similares (Tabela 2). Portanto, o valor do coeficiente de determinação obtido por padronização externa $(0,998)$ é satisfatório.

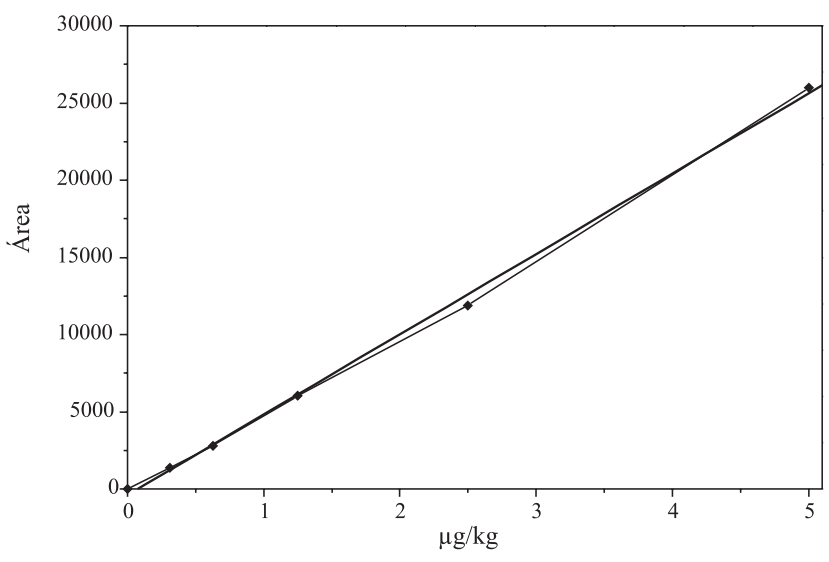

Figura 4. Curva analítica de CAP silanizado (área x concentração em $\mu \mathrm{g}$ ) kg) obtida por CG/DCE. $y=5200,3 x-382,43$ e $R^{2}=0,998$

\section{Precisão e exatidão}

A precisão do método foi determinada pela variação entre resultados obtidos em medidas sucessivas do teor de CAP no leite caprino fortificado, sendo avaliada pelo desvio padrão relativo (RSD), também chamado de coeficiente de variação (CV). ${ }^{34}$ Segundo o MERCOSUL e o Codex Alimentarius, para as faixas de concentrações analisadas o RSD indicado pode atingir $35 \%$ para concentrações menores que $1,0 \mu \mathrm{g} / \mathrm{kg}$, e até $30 \%$ para concentrações entre 1,0 e $10 \mu \mathrm{g} / \mathrm{kg} .{ }^{49,50}$ Para o parâmetro repetitividade o método apresentou-se preciso nos três níveis de concentração analisados, obtendo-se valores de RSD inferiores a $14 \%$ (Tabela 3).

Com relação à precisão intermediária (Tabela 4), os resultados entre diferentes dias e analistas foram analisados pelo tratamento estatístico ANOVA. Para os dois parâmetros o valor do $P$ foi maior que 0,05 , indicando que o método não apresentou diferença estatisticamente significativa entre as duas variáveis testadas com $95 \%$ de confiança.

A exatidão de um método mede quanto o resultado de um dado método analítico se aproxima do resultado real previamente definido. ${ }^{34}$ Para a validação do método de determinação de resíduo de CAP em leite de cabra, a exatidão foi medida através dos percentuais de recuperação do composto de interesse que foi adicionado na amostra nas concentrações de 0,30; 1,5 e 3,0 $\mu \mathrm{g} / \mathrm{kg}$. As recuperações médias obtidas variaram de 69,87 a 73,71\% (Tabela 3). Recuperações entre 60 e $90 \%$ são esperadas para métodos que envolvem etapas de derivatização, como as necessárias para a análise de CAP por CG, uma vez que podem acarretar maior perda do analito durante preparo da amostra, resultando em menor exatidão. ${ }^{23,24}$ Os percentuais de recuperação obtidos para os níveis de fortificação avaliados no método implantado estão de acordo com os limites estabelecidos nos critérios de aceitabilidade das normas. ${ }^{49,50}$ 
Tabela 2. Comparação entre os parâmetros de validação obtidos pelo presente método para leite caprino e os valores encontrados na literatura para leite bovino

\begin{tabular}{|c|c|c|c|c|c|c|c|c|}
\hline Ref. & $\begin{array}{c}\text { Técnica } \\
\text { Aplicada }\end{array}$ & Extração & Purificação & $\mathrm{R}^{2}$ & $\begin{array}{l}\mathrm{LOD} \\
(\mu \mathrm{g} / \mathrm{L})\end{array}$ & $\begin{array}{c}\mathrm{LOQ} \\
(\mu \mathrm{g} / \mathrm{L})\end{array}$ & $\begin{array}{c}\mathrm{CC} \alpha \\
(\mu \mathrm{g} / \mathrm{L})\end{array}$ & $\begin{array}{c}\mathrm{CC} \beta \\
(\mu \mathrm{g} / \mathrm{L}) \\
\end{array}$ \\
\hline Este estudo & $\mathrm{CG} / \mathrm{DCE}^{\mathrm{a}}$ & Acetato de etila & SPE C18 & 0,998 & 0,030 & 0,10 & & \\
\hline 24 & $\mathrm{CG} / \mathrm{DCE}^{\mathrm{a}}$ & Acetonitrila & SPE C18 & 0,9995 & 1,0 & 5,0 & & \\
\hline 23 & $\mathrm{CG} / \mathrm{EM} / \mathrm{EM}^{\mathrm{b}}$ & Acetonitrila & SPE C18 & 0,979 & & & 0,083 & 0,14 \\
\hline 26 & $\mathrm{CL} / \mathrm{EM} / \mathrm{EM}^{\mathrm{c}}$ & Acetonitrila & SPE C18 & 0,9605 & & & 0,11 & 0,15 \\
\hline 27 & $\mathrm{CL} / \mathrm{EM} / \mathrm{EM}^{\mathrm{c}}$ & Acetato de etila & Não utilizou & 0,998 & & & 0,090 & 0,11 \\
\hline 28 & $\mathrm{CL} / \mathrm{EM} / \mathrm{EM}^{\mathrm{c}}$ & Acetato de etila & Não utilizou & 0,980 & & & 0,050 & 0,090 \\
\hline 29 & $\mathrm{CL} / \mathrm{EM} / \mathrm{EM}^{\mathrm{c}}$ & Acetonitrila & Não utilizou & 0,999 & & & 0,020 & 0,040 \\
\hline 30 & $\mathrm{CL} / \mathrm{EM} / \mathrm{EM}^{\mathrm{c}}$ & Acido tricloroacético $10 \%$ & SPE HLB & 0,999 & & & 0,020 & 0,030 \\
\hline 31 & $\mathrm{CL} / \mathrm{EM} / \mathrm{EM}^{\mathrm{c}}$ & SPE C18 & Óxido Neutro de Alumínio & 0,9999 & & & - & 0,030 \\
\hline 39 & $\mathrm{CL} / \mathrm{EM}^{\mathrm{d}}$ & Tampão Fosfato pH 4.0 & $\mathrm{PMNE}^{\mathrm{e}}$ & 0,998 & 0,040 & 0,14 & & \\
\hline 48 & $\mathrm{CL} / \mathrm{EM}^{\mathrm{d}}$ & Acetonitrila & SPE LMS & 0,999 & 0,50 & 1,0 & & \\
\hline
\end{tabular}

${ }^{a}$ Cromatografia gasosa com detecção por captura de elétrons; ${ }^{\mathrm{b}}$ cromatografia gasosa com detecção por espectrometria de massas em tandem; ${ }^{\mathrm{c}}$ cromatografia líquida com detecção por espectrometria de massas em tandem; ${ }^{\mathrm{d}}$ cromatografia líquida com detecção por espectrometria de massas; ${ }^{\mathrm{e}}$ micro-extração com polímero monolítico; - dados não fornecidos

Tabela 3. Comparação entre os valores de exatidão e precisão intradia obtidos e os critérios de aceitação adotados ${ }^{49,50}$

\begin{tabular}{lcccc}
\hline $\begin{array}{l}\text { Nível de } \\
\text { fortificação } \\
(\mu \mathrm{g} / \mathrm{kg})\end{array}$ & $\begin{array}{c}\text { Exatidão } \\
\text { média } \\
(\%)\end{array}$ & $\begin{array}{c}\text { Precisão } \\
\text { intradia } \\
(\% \text { RSD })\end{array}$ & $\begin{array}{c}\text { Exatidão de } \\
\text { referência }^{49-50} \\
(\%)\end{array}$ & $\begin{array}{c}\text { Precisão de } \\
\text { referência }^{49-50} \\
(\% \text { RSD })\end{array}$ \\
\hline 0,30 & 73,71 & 6,54 & $50-120$ & 35 \\
1,5 & 69,87 & 5,80 & $60-120$ & 30 \\
3,0 & 70,40 & 13,40 & & \\
\hline
\end{tabular}

\section{Limites de detecção e quantificação}

O LOD determinado através da razão entre sinal:ruído (3:1) foi de $0,030 \mu \mathrm{g} / \mathrm{kg}$, e o LOQ determinado pela relação sinal:ruído (10:1) foi de $0,10 \mu \mathrm{g} / \mathrm{kg}$.

Métodos que utilizam as técnicas de CL/EM/EM e CG/EM/EM para a determinação de resíduos de CAP em leite bovino apresentam limites de decisão $(C C \alpha)$ e capacidades de detecção $(C C \beta)$ nas faixas de 0,020 a $0,11 \mu \mathrm{g} / \mathrm{kg}$ e de 0,030 a $0,15 \mu \mathrm{g} / \mathrm{kg}$, respectivamente (Tabela 2).23,26-31 Métodos utilizando a técnica de CL/EM também para a matriz leite bovino resultaram em um LOD entre 0,040 e $0,50 \mu \mathrm{g} / \mathrm{kg}$ e LOQ de 0,14 a 1,0 $\mu \mathrm{g} / \mathrm{kg} .{ }^{39,48}$ Vale ressaltar que estes métodos ${ }^{23,26-31,39,48}$ empregaram procedimentos de extrações diferentes dos utilizados no presente estudo, assim como etapas de purificação distintas ou ausentes (Tabela 2). Estas diferenças podem ter influenciado na recuperação do analito e, consequentemente, a sensibilidade dos métodos.

Portanto, nota-se que o emprego do CG/DCE nas condições utilizadas no presente método de validação permite atingir níveis de sensibilidade próximos a métodos que utilizam CL e CG associados com as técnicas de espectrometria de massas.

Comparando-se os dados de LOD e LOQ obtidos neste estudo com os dados da única referência encontrada que utilizou a técnica de CG/DCE para a determinação de CAP em leite bovino ${ }^{24}$ (Tabela 2), observa-se que os LOD e LOQ obtidos no presente estudo $(0,030$ e $0,10 \mu \mathrm{g} / \mathrm{kg}$ ) são cerca de 30 vezes maiores para o LOD e 50 vezes maiores para o LOQ. Uma vez que as matrizes de leites bovinos e caprinos possuem semelhanças na composição centesimal, ${ }^{57}$ esta diferença de sensibilidade pode ser devida aos distintos procedi-
Tabela 4. Avaliação da precisão intermediária pelas variáveis "dia" e "analista"

\begin{tabular}{lccc}
\hline Variáveis & & Média (\%RSD) & $P^{*}$ \\
\hline \multirow{2}{*}{ Dia } & 1 & 73,71 & 0,617965 \\
& 2 & 70,41 & \\
\multirow{2}{*}{ Analista } & 1 & 70,41 & 0,93223 \\
& 2 & 69,87 & \\
\hline${ }^{*}$ P $>0,05-$ Sem diferenca significativa. & &
\end{tabular}

mentos de extração das amostras, tais como, não remoção da fração lipídica, ${ }^{24}$ que pode resultar em um aumento no nível de interferentes e, a utilização de água para retomar o extrato seco anterior à etapa de purificação por SPE-C $18,{ }^{24}$ que pode ter reduzido a recuperação do resíduo e a sensibilidade do método, considerando que o CAP tem baixa solubilidade neste solvente..$^{58}$

\section{Robustez}

Um método robusto tem a habilidade de fornecer resultados inalterados quando sujeito a pequenas mudanças. Portanto, uma vez detectado que a massa de sulfato de sódio estava diretamente relacionada ao percentual de separação de acetato de etila utilizado durante a extração do CAP, esta variável foi escolhida para avaliar a robustez. A outra variável escolhida foi o tempo de derivatização, devido ao método de referência ${ }^{22} \mathrm{e}$ a instrução de uso do reagente de silanização fornecerem informações diferentes. Pela análise estatística dos resultados, foi verificado que o valor obtido para o $F$ calculado foi maior que o $F$ tabelado para a variável massa de sulfato de sódio, demonstrando que este é um ponto crítico para o método proposto de determinação de resíduo de CAP em leite de cabra (Tabela 5). Para a variável tempo de derivatização, o valor obtido para o $F$ calculado foi inferior ao do $F$ tabelado, determinando com $95 \%$ de confiança que o método é robusto para esta variável (Tabela 5).

Os resultados da presente validação para a quantificação por CG/ DCE de resíduos de CAP em leite caprino mostraram que os parâmetros de validação (seletividade, linearidade, exatidão, precisão, LOD, LOQ e robustez) são similares aos obtidos por métodos que usam as técnicas $\mathrm{CL} / \mathrm{EM} / \mathrm{EM}$ e CG/EM/EM para leite bovino. O método proposto reduz 
os custos e a demanda de tempo da análise por utilizar padronização externa. Além disso, deve-se considerar que a técnica de CG/DCE é mais amplamente difundida nos laboratórios de análises, reduzindo a necessidade de investimentos para aquisição de novos equipamentos e para treinamento de profissionais. O método desenvolvido por CG/DCE para a determinação de resíduos de CAP em leite caprino possui sensibilidade compatível com as técnicas de CL/EM/EM e CG/EM/EM para leite bovino, sendo uma técnica altamente sensível, podendo ser utilizado como uma alternativa para o monitoramento e/ou screening deste analito.

Tabela 5. Avaliação estatística da robustez pelas variáveis "massa de $\mathrm{Na}_{2} \mathrm{SO}_{4}$ " e "tempo de derivatização"

\begin{tabular}{lcccc}
\hline Variáveis & & $\begin{array}{c}\text { Recuperação } \\
\text { média }(\%)\end{array}$ & $\begin{array}{c}F \\
\text { calculado }\end{array}$ & $\begin{array}{c}F \\
\text { tabelado }\end{array}$ \\
\hline Massa de $\mathrm{Na}_{2} \mathrm{SO}_{4}$ & 7 & 72,53 & & \\
$(\mathrm{~g} \pm 0,1)$ & 10 & 73,71 & 27,30801 & \\
& 13 & 55,23 & & 5,143253 \\
Tempo de de- & 10 & 74,62 & & \\
rivatização (min) & 15 & 73,71 & 0,304138 & \\
& 20 & 72,29 & & \\
\hline
\end{tabular}

\section{CONCLUSÃO}

Foi validado intralaboratorialmente um método alternativo utilizando a técnica de CG/DCE para determinação quantitativa de resíduo de cloranfenicol em matriz de leite caprino. O procedimento analítico proposto mostrou-se preciso, exato, atendendo aos critérios de aceitabilidade estabelecidos pelo MERCOSUL e Codex Alimentarius. O método proposto utiliza a vantagem da alta sensibilidade do DCE para compostos halogenados, sendo uma alternativa para monitoramento e/ou screening de resíduos de CAP em leite caprino para laboratórios que não possuem as técnicas CL/EM/EM ou CG/ EM/EM, de alto custo de aquisição.

\section{AGRADECIMENTOS}

À FAPESB, CAPES e ao Banco do Nordeste do Brasil, pelo apoio financeiro.

\section{REFERÊNCIAS}

1. Agência Nacional de Vigilância Sanitária (ANVISA); Programa Nacional de Análise de Resíduos de Medicamentos Veterinários em Alimentos Expostos ao Consumo (PAMVet), 2003.

2. European Commission; Official Journal of the European Union L71, 17, 2003.

3. Santos, S. M.; Henriques, M.; Duarte, A. C.; Esteves, V. I.; Talanta 2007, 71,731 .

4. Gatica, C. P.; Gesche, E. R.; Rev. Científica (Maracaíbo) 2007, 17, 231.

5. United States Food and Drug Administration (USFDA); Laboratory Information Bulletin (LIB) $\mathrm{n}^{\circ}$ 4290, 2002.

6. Akhtar, M. H.; Danis, C.; Sauve, A.; Barry, C.; J. Chromatogr., A 1995, 696, 123.

7. Keukens, H. J.; Beek, W. M.; Aerts, M. M.; J. Chromatogr., A 1986, 352, 445 .

8. http://www.medscape.com, acessada em Novembro 2008.

9. World Health Organization (WHO); Joint FAO/WHO Expert Committee on Food Additives, sixty-second meeting, February, 2004; http://jecfa. ilsi.org/evaluation.cfm?chemical=CHLORAMPHENICOL, acessada em Maio 2009.
10. Shitandi, A.; Sternesjö, A.; J. Dairy Sci. 2004, 87, 4145; Schlegelova, J.; Babak, V.; Klimova, E.; Lukasova, J.; Navratilova, P.; Sustackova, A.; Sediva, I.; Rysanek, D.; J. Vet. Med. B 2002, 49, 216; Perreten, V.; Giampa, N.; Schmid, U. S.; Teuber, M.; Syst. Appl. Microbiol. 1998, 21, 113; Ombui, J. N.; Kimotho, A. M.; Nduhiu, J. G.; East Afr. Med. J. 2000, 77, 463; Vasil, M.; Vet. Med. 1999, 44, 115; Araújo, V. J. A.; Monografia, Universidade Federal de Campina Grande, Brasil, 2008; Alomirah, H.; Al-Mazeedi, H.; Al-Zenki, S.; Al-Aati, T.; Al-Otaibi, J.; Al-Batel, M.; Sidhu, J.; J. Food Qual. 2007, 30, 745.

11. Siqueira, I. N.; Dissertação de Mestrado, Universidade Federal de Campina Grande, Brasil, 2007.

12. Fracalanzza, S. A. P.; Scheidegger, E. M. D.; Santos, P. F.; Leite, P. C.; Teixeira, L. M.; Mem. Inst. Oswaldo Cruz, 2007, 102, 853.

13. Tendencia, E. A.; Pena, L. D.; Aquaculture 2001, 195, 193.

14. Instituto Brasileiro de Geografia e Estatística (IBGE); Censo Agropecuário de 2007, 2007.

15. http://www.fao.org, acessada em Dezembro 2008.

16. Ribeiro, E. L. A.; Ribeiro, H. J. S. S.; Semina: Ciências Agrárias 2001, $22,229$.

17. Santos, U.; Ramos, F.; Curr. Pharm. Anal. 2006, 2, 53; Brasil, Ministério da Agricultura, Pecuária e Abastecimento (MAPA); Portaria no 448, de 10/09/1998; Commission Regulation; Official Journal of the European Communities, L142, 20, 1998; Food and Drug Administration (FDA); National Drug Residue Milk Monitoring Program, 7303.039, 1997; Brasil, Agência Nacional de Vigilância Sanitária (ANVISA), Resolução RDC n ${ }^{\circ} 253$, de 16/09/2003.

18. Ministério da Agricultura, Pecuária e Abastecimento (MAPA); Método $n^{\circ} 203 / 01,2002$

19. Lee, J. B.; Chung, H. H.; Chung, Y. H.; Lee, K. G.; Food Chem. 2007, 105,1726

20. Perez, N.; Gutierrez, R.; Noa, M.; Diaz, G.; Luna, H.; Escobar, I.; Muniye, Z.; J. AOAC Int. 2002, 85, 20.

21. Shi, X.; Wu, A.; Zheng, S.; Li, R.; Zhang, D.; J. Chromatogr., B: Anal. Technol. Biomed. Life Sci. 2007, 850, 24; Moretti, V. M.; Vandewater, C.; Haagsma, N.; J. Chromatogr., B: Anal. Technol. Biomed. Life Sci. 1992, 583, 77; Long, A. R.; Hsieh, L. C.; Bello, A. C.; Malbrough, M. S.; Short, C. R.; Barker, S. A.; J. Agric. Food Chem. 1990, 38, 427.

22. Ministério da Agricultura, Pecuária e Abastecimento (MAPA); Método $n^{\circ}$ 203/03, 1999 .

23. Sniegocki, T.; Posyniak, A.; Zmudzki, J.; Bull. Vet. Institute in Pulawy 2006, $50,353$.

24. Pfenning, A. P.; Madson, M. R.; Roybal, J. E.; Turnipseed, S. B.; Gonzales, S. A.; Hurlbut, J. Á.; Salmon, G. D.; J. AOAC Int. 1998, 81, 714.

25. United States Food and Drug Administration (USFDA); Laboratory Information Bulletin (LIB) $\mathrm{n}^{\circ}$ 4306, 2003.

26. Sniegocki, T.; Posyniak, A.; Zmudzki, J.; Bull. Vet. Institute in Pulawy 2007, 51, 59 .

27. Rodziewicz, L.; Zawadzka, I.; Talanta 2008, 75, 846.

28. Nicolich, R. S.; Barroso, E. W.; Marques, M. A. S.; Anal. Chim. Acta 2006, $565,97$.

29. Ronning, H. T.; Einarsen, K.; Asp, T. N.; J. Chromatogr., A 2006, 1118, 226.

30. Guy, P. A.; Royer, D.; Mottier, P.; Gremaud, E.; Perisset, A.; Stadler, R. H.; J. Chromatogr., A 2004, 1054, 365.

31. Sorensen, L. K.; Elbaek, T. H.; Hansen, H.; J. AOAC Int. 2003, 86, 703.

32. Vieira, E.; Lichtig, J.; Arq. Instituto Biológico 2004, 71, 698.

33. Brasil, Agencia Nacional de Vigilância Sanitária (ANVISA); Resolução RE no 899, de 29/05/2003.

34. Ribani, M.; Melo, L. F. C.; Bottoli, C. B. G.; Collins, C. H.; Jardim, I. C. S. F.; Quim. Nova 2004, 27, 771.

35. Lanças, F. M.; Validação de Métodos Cromatográficos de Análise, RIMA: São Carlos, 2004.

36. Pezza, L.; Rios, A.; Nozal, L.; Arce, L.; Valcarcel, M.; Quim. Nova 2006, $29,926$. 
37. Song, W. W.; Ding, M. X.; Zhang, N. W.; Liu, H. F.; Xu, M. G.; Liu, G. Y.; Chai, C. Y.; Chin. J. Anal. Chem. 2007, 35, 1731.

38. Chai, C.; Xu, M.; Liu, G.; Chin. J. Anal. Chem. 2006, 34, 1715.

39. Huang, J. F.; Zhang, H. J.; Feng, Y. Q.; J. Agric. Food Chem. 2006, 54, 9279.

40. Ferguson, J.; Baxter, A.;Young, P.; Kennedy, G.; Elliott, C.; Weigel, S.; Gatermann, R.; Ashwin, H.; Stead, S.; Sharman, M.; Anal. Chim. Acta 2005, 529, 109.

41. Lin, S.; Han, S. Q.; Liu, Y. B.; Guan, W. G. X. G. Y.; Anal. Bioanal. Chem. 2005, 382, 1250 .

42. Scortichini, G.; Annunziata, L.; Haouet, M. N.; Benedetti, F.; Krusteva, I.; Galarini, R.; Anal. Chim. Acta 2005, 535, 43.

43. Althaus, R. L.; Torres, A.; Montero, A.; Balasch, S.; Molina, M. P.; J. Dairy Sci. 2003, 86, 457.

44. Ramirez, A.; Gutierrez, R.; Diaz, G.; Gonzalez, C.; Perez, N.; Vega, S.; Noa, M.; J. Chromatogr., B: Anal. Technol. Biomed. Life Sci. 2003, 784, 315 .

45. Agui, L.; Guzman, A.; Sedeno,Y.; Pingarron, J. M.; Anal. Chim. Acta 2002, 461, 65

46. Althaus, R.; Peris, C.; Montero, A.; Torres, A.; Molina, P.; Milchwissenschaft 2002, 57, 660.

47. Gaudin, V.; Maris, P.; Food Agr. Immunol. 2001, 13, 77.

48. Hormazabal, V.; Yndestad, M.; J. Liq. Chromatogr. Relat. Technol. 2001, 24, 2477.
49. Mercosul, Mercado Comum do Cone Sul; Resolução 57, 1994.

50. Codex Alimentarius; Residuos de medicamentos veterinarios en los alimentos, Roma, 1993.

51. United States Department of Agriculture (USDA); CAM Method 5.0223, 1991 .

52. United States Department of Agriculture (USDA); Methods of analysis for veterinary drug residues: validation guidelines, 1998.

53. Agência Nacional de Vigilância Sanitária (ANVISA); Relatório 2004/2005-Monitoramento de resíduos em leite exposto ao consumo, 2006 .

54. Siqueira, S. R. R.; Dissertação de Mestrado, Universidade Estadual de Campinas, Brasil, 2007.

55. Li, T. L.; Chung-wang, Y. J.; Shih, Y. C.; J. Food Sci. 2001, 67, 21.

56. Gantverg, A.; Shishani, I.; Hoffmann, M.; Anal. Chim. Acta 2003, 483, 125.

57. Torres, E. A. F. S.; Campos, N. C.; Duarte, M.; Garbelotti, M. L.; Philippi, S. T.; Minazzi-Rodrigues, R. S.; Ciênc. Tecnol. Aliment. 2000, 20, 145; Prata, L. F.; Ribeiro, A. C.; Rezende, K. T.; Carvalho, M. R. B.; Ribeiro, S. D. A.; Costa, R. G.; Ciênc. Tecnol. Aliment. 1998, 18, 428.

58. Sigma; Product Information: Chloramphenicol, 1999; http://www. medscape.com, acessada em Janeiro 2009 